PROCEEDINGS OF THE

AMERICAN MATHEMATICAL SOCIETY

Volume 126, Number 10, October 1998, Pages 3037-3044

S 0002-9939(98)04483-9

\title{
A THEOREM ON ITERATIONS OF POLYNOMIAL MAPS IN SEVERAL VARIABLES
}

\author{
NILSON C. BERNARDES JR.
}

(Communicated by Theodore W. Gamelin)

\begin{abstract}
We establish a polynomial version of a theorem obtained by Enflo, Gurarii, Lomonosov and Lyubich for linear operators. As a consequence, we also derive a polynomial version of a result due to Pták.
\end{abstract}

\section{INTRODUCTION}

Throughout this paper $\mathbf{K}$ denotes either the field $\mathbf{R}$ of real numbers or the field $\mathbf{C}$ of complex numbers, $\mathbf{N}$ denotes the set of non-negative integers and $\mathbf{N}^{*}=\mathbf{N}-\{0\}$. We shall always assume $\mathbf{K}^{n}\left(n \in \mathbf{N}^{*}\right)$ endowed with its euclidean norm, and if $P: \mathbf{K}^{n} \rightarrow \mathbf{K}^{n}$ is a homogeneous polynomial map we consider

$$
\|P\|=\sup _{\|x\| \leq 1}\|P x\| .
$$

If $X$ and $Y$ are sets, then the relation " $X \supset Y$ " means that $X$ contains $Y$ properly and the relation " $X \supseteq Y$ " means that $X$ contains or is equal to $Y$. Moreover, we denote by $X-Y$ the difference set $\{x \in X ; x \notin Y\}$.

Pták [4] established the following result:

If $T: \mathbf{K}^{n} \rightarrow \mathbf{K}^{n}$ is a linear operator which satisfies $1=\|T\|=\ldots=\left\|T^{n}\right\|$, then $\left\|T^{j}\right\|=1$ for all $j \geq 1$.

Later, Enflo, Gurarii, Lomonosov and Lyubich [1] obtained the following pointwise version of Pták's result :

There is a constant $C=C_{\mathbf{K}}(n) \in \mathbf{N}^{*}$ such that for any linear operator $T$ : $\mathbf{K}^{n} \rightarrow \mathbf{K}^{n}$ and any $x \in \mathbf{K}^{n}$, the relation $1=\|x\|=\|T x\|=\ldots=\left\|T^{C} x\right\|$ implies $\left\|T^{j} x\right\|=1$ for all $j \geq 1$.

The goal of the present paper is to obtain polynomial versions of the above mentioned theorems. Our main result is the following:

Theorem 1. Let $n$ and $d$ be positive integers. Then there is a constant $M=$ $M_{\mathbf{K}}(n, d) \in \mathbf{N}^{*}$ such that for any homogeneous polynomial map $P: \mathbf{K}^{n} \rightarrow \mathbf{K}^{n}$ of degree $d$ and any $x \in \mathbf{K}^{n}$, the relation

$$
1=\|x\|=\|P x\|=\ldots=\left\|P^{M} x\right\|
$$

implies

$$
\left\|P^{j} x\right\|=1 \text { for all } j \geq 1
$$

Received by the editors March 15, 1997.

1991 Mathematics Subject Classification. Primary 32H50, 26B99.

(C)1998 American Mathematical Society 
As an immediate consequence of this theorem, we have the following

Theorem 2. Let $n$ and $d$ be positive integers. Then there is a constant $N=$ $N_{\mathbf{K}}(n, d) \in \mathbf{N}^{*}$ such that for any homogeneous polynomial map $P: \mathbf{K}^{n} \rightarrow \mathbf{K}^{n}$ of degree $d$, the relation $1=\|P\|=\ldots=\left\|P^{N}\right\|$ implies $\left\|P^{j}\right\|=1$ for all $j \geq 1$.

In the next section we prove theorem 1 modulo two lemmas. These lemmas will be proved in $\S 3$. Finally, in $\S 4$ we show that the argument used to prove theorem 1 can also be applied to establish other results of similar type.

\section{Proof of theorem 1}

Without loss of generality, we may assume $\mathbf{K}=\mathbf{R}$. Put

$$
\alpha_{j}=2^{j} d^{1+2+\ldots+j} \quad(j \geq 1) .
$$

We consider a set $B$ of the form

$$
B=\left\{b_{i_{0}, \ldots, i_{r}} ; r \in \mathbf{N} \text { and } i_{0}, \ldots, i_{r} \in \mathbf{N}^{*}\right\},
$$

where the $b_{i_{0}, \ldots, i_{r}}$ 's are distinct objects. The elements of $B$ will be called pieces. We define a relation " $>$ " on $B$ by

$$
b_{i_{0}, \ldots, i_{r}}>b_{j_{0}, \ldots, j_{s}} \text { if and only if } s=r+1 \text { and } j_{0}=i_{0}, \ldots, j_{r}=i_{r} .
$$

If $b, b^{\prime} \in B$ and $b>b^{\prime}$, we say that $b^{\prime}$ is a subpiece of $b$. We also consider the collection $\Omega$ of all finite sequences

$$
S=\left(B_{1}, \ldots, B_{r}\right)
$$

of subsets of $B$ with the following properties:

(a) $B_{1} \subset\left\{b_{i} ; i \in \mathbf{N}^{*}\right\}$;

(b) Each $B_{j}$ has at most $\alpha_{j}$ pieces;

(c) For each $j \in\{2, \ldots, r\}$ and for each piece $x$ of $B_{j}$, either $x$ is a piece of $B_{j-1}$ or $x$ is a subpiece of some piece $y$ of $B_{j-1}$; moreover, in the latter case, we cannot have $y \in B_{j}$;

(d) $B_{1} \neq B_{2} \neq \ldots \neq B_{r}$

(e) There cannot exist integers $1 \leq i_{0}<\ldots<i_{n} \leq r$ and pieces $x_{i_{0}} \in B_{i_{0}}, \ldots, x_{i_{n}} \in$ $B_{i_{n}}$ so that $x_{i_{0}}>x_{i_{1}}>\ldots>x_{i_{n}}$.

We claim that the following holds :

There is a constant $M=M(n, d) \in \mathbf{N}^{*}$ such that every sequence in $\Omega$ has length $\leq M$, where the length of a sequence $S=\left(B_{1}, \ldots, B_{r}\right) \in \Omega$ is defined to be the number $r$.

Assume (3) for the moment, and let us see how it can be used to prove theorem 1. For each $j \in \mathbf{N}^{*}$, let $q_{j}$ be the real homogeneous polynomial given by

$$
q_{j}(y)=\left\langle P^{j} y, P^{j} y\right\rangle-\langle y, y\rangle^{d^{j}} \quad\left(y \in \mathbf{R}^{n}\right),
$$

where $\langle\cdot, \cdot\rangle$ denotes the scalar product on $\mathbf{R}^{n}$. Define

$$
\begin{aligned}
A_{j} & =\left\{y \in \mathbf{R}^{n} ; q_{k}(y)=0 \text { for } k=1, \ldots, j\right\} \\
& =\left\{y \in \mathbf{R}^{n} ;\left\|P^{k} y\right\|=\|y\|^{d^{k}} \text { for } k=1, \ldots, j\right\},
\end{aligned}
$$

for $j \geq 1$. Obviously,

$$
A_{1} \supseteq A_{2} \supseteq A_{3} \supseteq \cdots .
$$


For each $j \in \mathbf{N}^{*}$, consider the algebraic subset

$$
Y_{j}=Z\left(\tilde{q}_{1}\right) \cap \ldots \cap Z\left(\tilde{q}_{j}\right)
$$

of the projective $(n-1)$-space $\mathbf{P}^{n-1}$ over $\mathbf{C}$ (where $\tilde{q}_{j}$ denotes the polynomial $q_{j}$ regarded as a complex polynomial in $n$ complex variables and $Z\left(\tilde{q}_{j}\right)$ is the zero set of $\tilde{q}_{j}$ in $\mathbf{P}^{n-1}$; see [3]). Obviously, $Y_{1} \supseteq Y_{2} \supseteq \cdots$. Since $\mathbf{P}^{n-1}$ is a Noetherian topological space, there is a greatest integer $\gamma$ such that

$$
Y_{1} \supset Y_{2} \supset \ldots \supset Y_{\gamma} \text {. }
$$

Now we need the following:

Lemma 3. There is a sequence in $\Omega$ with length $\gamma$.

The proofs of this and the next lemma will be left to the next section.

By (3) and lemma $3, \gamma \leq M$. Our definition of $\gamma$ implies that $Y_{\gamma}=Y_{\gamma+1}$. Hence, if $y \in A_{\gamma}-\{0\}$ then

$$
\phi(y) \in Z\left(\tilde{q}_{1}\right) \cap \ldots \cap Z\left(\tilde{q}_{\gamma}\right)=Y_{\gamma}=Y_{\gamma+1} \subseteq Z\left(\tilde{q}_{\gamma+1}\right)
$$

(where $\phi(y)$ denotes the equivalence class of $y$ in $\mathbf{P}^{n-1}$ ), which implies $y \in A_{\gamma+1}$. Thus, $A_{\gamma}=A_{\gamma+1}$. Now, if $y \in A_{\gamma+1}$ then $P y \in A_{\gamma}$ and so $P y \in A_{\gamma+1}$, which implies $y \in A_{\gamma+2}$. Hence, $A_{\gamma+1}=A_{\gamma+2}$. By induction, we see that $A_{\gamma}=A_{\gamma+1}=$ $A_{\gamma+2}=\cdots$. In particular,

$$
A_{M}=A_{M+1}=A_{M+2}=\cdots .
$$

If $x$ satisfies (1), then $x \in A_{M}$ and so $x \in A_{j}$ for all $j \geq M$, which gives (2). This proves theorem 1.

We shall establish (3) by presenting an algorithm that constructs a longest possible sequence in $\Omega$. The main motivation for the algorithm is based on the following observation: By (c), we can think of $B_{j}$ as obtained from $B_{j-1}$ by repeating or deleting or replacing by subpieces each of the pieces of $B_{j-1}$.

First, let us make some definitions. Let $S=\left(B_{1}, \ldots, B_{r}\right) \in \Omega$. If $x \in B_{j}$ for some $j \in\{1, \ldots, r\}$, say $x=b_{j_{0}, \ldots, j_{m}}$, we define the depth of $x$ (denoted $\operatorname{depth}(x)$ ) as being the number $m$. Note that this is the largest integer with the property that we can find integers $1 \leq i_{0}<\ldots<i_{m}=j$ and pieces $x_{i_{0}} \in B_{i_{0}}, \ldots, x_{i_{m}} \in B_{i_{m}}$ so that

$$
x_{i_{0}}>x_{i_{1}}>\ldots>x_{i_{m}}=x .
$$

With this definition we see that condition (e) merely says that each piece in each $B_{j}$ must have depth $\leq n-1$. We also define the numbers

$$
N_{i}(S, j)=\operatorname{card}\left\{x \in B_{j} ; \operatorname{depth}(x)=i\right\} \quad(i=0, \ldots, n-1 ; j=1, \ldots, r)
$$

and put

$$
N(S, j)=\left(N_{0}(S, j), \ldots, N_{n-1}(S, j)\right) \quad(j=1, \ldots, r) .
$$

Note that $N(S, j) \in \mathbf{N}^{n}$. In the sequel we consider $\mathbf{N}^{n}$ endowed with its lexicographic order relation, which we denote by " $\leq$ ".

Algorithm. 1. Put $\tilde{B}_{1}=\left\{b_{1}, \ldots, b_{\alpha_{1}}\right\}$.

2. While $\tilde{B}_{j} \neq \emptyset$ do:

- if $\tilde{B}_{j}$ has a piece of depth $n-1$, choose one such piece $x$ and define $\tilde{B}_{j+1}=\tilde{B}_{j}-\{x\}$; 
- if $\tilde{B}_{j}$ has no piece of depth $n-1$, choose a piece $x$ of $\tilde{B}_{j}$ with maximum depth. Then choose $\alpha_{j+1}-\operatorname{card}\left(\tilde{B}_{j}\right)+1$ subpieces $x_{1}, \ldots, x_{\alpha_{j+1}-\operatorname{card}\left(\tilde{B}_{j}\right)+1}$ of $x$ and define $\tilde{B}_{j+1}=\left(\tilde{B}_{j}-\{x\}\right) \cup\left\{x_{1}, \ldots, x_{\alpha_{j+1}-\operatorname{card}\left(\tilde{B}_{j}\right)+1}\right\}$.

3. If $\tilde{B}_{j}=\emptyset$, stop.

Let $\tilde{S}=\left(\tilde{B}_{1}, \tilde{B}_{2}, \ldots\right)$ be the sequence generated by our algorithm. Clearly our algorithm never generates pieces of depth $\geq n$, and in each step it decreases the depth numbers in lexicographic order: $N(\tilde{S}, j+1)<N(\tilde{S}, j)$. Since the lexicographic order relation on $\mathbf{N}^{n}$ is a well-order, it follows that our algorithm must end in a finite number of steps, so that $\tilde{S}$ is a finite sequence; say

$$
\tilde{S}=\left(\tilde{B}_{1}, \ldots, \tilde{B}_{M}\right) \text {. }
$$

Moreover, it is immediate to check that $\tilde{S} \in \Omega$. Now, we will need the fact that our sequence $\tilde{S}$ has "maximal depth numbers":

Lemma 4. If $S=\left(B_{1}, \ldots, B_{r}\right)$ is any element of $\Omega$, then

$$
N(\tilde{S}, j) \geq N(S, j) \quad \text { for } \text { all } j=1, \ldots, \min \{M, r\} .
$$

Let us finally prove (3). We claim that for any sequence $S=\left(B_{1}, \ldots, B_{r}\right) \in \Omega$, we have $r \leq M$. Suppose that this is not the case, and let $S \in \Omega$ be a sequence with length $r>M$. By lemma $4, N(S, M) \leq N(\tilde{S}, M)$. Since $\tilde{B}_{M}=\emptyset$, we have $N(\tilde{S}, M)=(0, \ldots, 0)$. Hence, $N(S, M)=(0, \ldots, 0)$, which implies $B_{M}=\emptyset$. It now follows from (c) that $B_{M+1}=\emptyset$. Hence, $B_{M}=B_{M+1}$, which contradicts (d).

\section{Proofs of the Lemmas}

Proof of lemma 3. For each $j \in\{1, \ldots, \gamma\}$, let $C_{j}$ be the set of all irreducible components of $Y_{j}$. Since each $\tilde{q}_{j}$ has degree $\leq 2 d^{j}$, it follows that

$$
\operatorname{card}\left(C_{j}\right) \leq(2 d)\left(2 d^{2}\right) \ldots\left(2 d^{j}\right)=\alpha_{j} \quad \text { for } j=1, \ldots, \gamma
$$

([2], example 8.4.6). For each $j \in\{1, \ldots, \gamma\}$ and each $Z \in C_{j}$, we define the depth of $Z$ (denoted $\operatorname{depth}(Z)$ ) as the greatest integer $m \in \mathbf{N}$ such that we can find integers $1 \leq i_{0}<\ldots<i_{m}=j$ and elements $Z_{i_{0}} \in C_{i_{0}}, \ldots, Z_{i_{m}} \in C_{i_{m}}$ so that

$$
Z_{i_{0}} \supset Z_{i_{1}} \supset \ldots \supset Z_{i_{m}}=Z \text {. }
$$

Note that since $\mathbf{P}^{n-1}$ has dimension $n-1$ [3], each element of each $C_{j}$ must have depth $\leq n-1$. We shall construct, inductively, subsets $B_{1}, \ldots, B_{\gamma}$ of $B$ and bijections $\phi_{1}: C_{1} \rightarrow B_{1}, \ldots, \phi_{\gamma}: C_{\gamma} \rightarrow B_{\gamma}$, so that

$$
\left(B_{1}, \ldots, B_{j}\right) \in \Omega
$$

and

$$
\operatorname{depth}\left(\phi_{j}(Z)\right) \leq \operatorname{depth}(Z) \text { for all } Z \in C_{j},
$$

for $j=1, \ldots, \gamma$. This will prove the lemma.

We begin by putting $B_{1}=\left\{b_{1}, \ldots, b_{\operatorname{card}\left(C_{1}\right)}\right\}$ and by choosing an arbitrary bijection $\phi_{1}: C_{1} \rightarrow B_{1}$. It is clear that (4) and (5) hold for $j=1$. Suppose that for some $t \in\{1, \ldots, \gamma-1\}$ we have already constructed $B_{1}, \ldots, B_{t} \subset B$ and bijections $\phi_{1}: C_{1} \rightarrow B_{1}, \ldots, \phi_{t}: C_{t} \rightarrow B_{t}$ so that (4) and (5) hold for $j=1, \ldots, t$. Since $Y_{t} \supset Y_{t+1}$, each element of $C_{t+1}$ is either an element of $C_{t}$ or a proper subset of some element of $C_{t}$. Let $Z_{1}, \ldots, Z_{\beta}$ be the elements of $C_{t+1} \cap C_{t}$. We define

$$
\phi_{t+1}\left(Z_{i}\right)=\phi_{t}\left(Z_{i}\right) \quad \text { for } i=1, \ldots, \beta \text {. }
$$


Let $W_{1}, \ldots, W_{\alpha}$ be an enumeration of the elements of $C_{t}-C_{t+1}$. Let $W_{1,1}, \ldots, W_{1, \beta_{1}}$ be the elements of $C_{t+1}$ that are proper subsets of $W_{1}$. By definition, $\phi_{t}\left(W_{1}\right) \in B$, say $\phi_{t}\left(W_{1}\right)=b_{i_{1}, \ldots, i_{s}}$. We then define

$$
\phi_{t+1}\left(W_{1, i}\right)=b_{i_{1}, \ldots, i_{s}, i} \quad \text { for } i=1, \ldots, \beta_{1} .
$$

Now, let $W_{2,1}, \ldots, W_{2, \beta_{2}}$ be the elements of $C_{t+1}-\left\{W_{1,1}, \ldots, W_{1, \beta_{1}}\right\}$ that are proper subsets of $W_{2}$. If $\phi_{t}\left(W_{2}\right)=b_{j_{1}, \ldots, j_{w}}$, we then define

$$
\phi_{t+1}\left(W_{2, i}\right)=b_{j_{1}, \ldots, j_{w}, i} \quad \text { for } i=1, \ldots, \beta_{2} .
$$

By continuing this process, we obtain a function $\phi_{t+1}: C_{t+1} \rightarrow B$. Put

$$
B_{t+1}=\phi_{t+1}\left(C_{t+1}\right) \text {. }
$$

The main ingredient for checking that $B_{t+1}$ and $\phi_{t+1}$ have the desired properties is the fact that for any $S^{\prime}=\left(B_{1}^{\prime}, \ldots, B_{r}^{\prime}\right) \in \Omega$ no two pieces of any $B_{j}^{\prime}$ can be comparable under ">" (which can be easily proved by induction on $j$ ). We leave the details to the reader.

Proof of lemma 4. We proceed by induction on $j$. If $j=1$ then

$$
N(\tilde{S}, 1)=\left(\alpha_{1}, 0,0, \ldots, 0\right) \geq N(S, 1) .
$$

Suppose that for a certain $j \in\{1, \ldots, \min \{M, r\}-1\}$ we have $N(\tilde{S}, j) \geq N(S, j)$. We have to show that

$$
N(\tilde{S}, j+1) \geq N(S, j+1)
$$

We have two cases:

Case 1. $N(\tilde{S}, j)=N(S, j)$ : This means that

$$
N_{i}(\tilde{S}, j)=N_{i}(S, j) \quad \text { for } i=0, \ldots, n-1 .
$$

Let $\beta$ be the maximum depth of the pieces of $\tilde{B}_{j}$, so that

$$
N_{\beta+1}(\tilde{S}, j)=\ldots=N_{n-1}(\tilde{S}, j)=0 .
$$

By our construction of $\tilde{B}_{j+1}$,

$$
N_{i}(\tilde{S}, j+1)=N_{i}(\tilde{S}, j)=N_{i}(S, j) \quad \text { for } i=0, \ldots, \beta-1 .
$$

Suppose that for some $i \in\{1, \ldots, \beta\}$ we have $N_{i}(S, j+1)>N_{i}(S, j)$. Then, in the construction of $B_{j+1}$, at least one piece of $B_{j}$ with depth $i-1$ must have been replaced by subpieces. Let $i_{0} \in\{0, \ldots, \beta-1\}$ be the smallest integer such that some piece of $B_{j}$ with depth $i_{0}$ was replaced by subpieces. Then, $N_{i}(\tilde{S}, j+1)=N_{i}(S, j) \geq$ $N_{i}(S, j+1)$ for $i=0, \ldots, i_{0}-1$, and $N_{i_{0}}(\tilde{S}, j+1)=N_{i_{0}}(S, j)>N_{i_{0}}(S, j+1)$ (by (7)), and so (6) holds. Thus, assume that

$$
N_{i}(S, j+1) \leq N_{i}(S, j) \quad \text { for } i=1, \ldots, \beta .
$$

If for some $i \in\{0, \ldots, \beta-1\}$ we have $N_{i}(S, j+1)<N_{i}(S, j)$, then (6) holds (by (7)). Hence, assume

$$
N_{i}(S, j+1)=N_{i}(S, j) \quad \text { for } i=0, \ldots, \beta-1 .
$$

By (7) and (8), we obtain

$$
N_{i}(\tilde{S}, j+1)=N_{i}(S, j+1) \quad \text { for } i=0, \ldots, \beta-1 .
$$

By (8), in the construction of $B_{j+1}$, no piece of $B_{j}$ with depth $\leq \beta-1$ was removed nor replaced by subpieces. Since $N_{i}(S, j)=N_{i}(\tilde{S}, j)=0$ for $j=\beta+1, \ldots, n-1$, 
some piece of $B_{j}$ with depth $\beta$ must have been removed or replaced by subpieces, so that $N_{\beta}(S, j+1) \leq N_{\beta}(S, j)-1=N_{\beta}(\tilde{S}, j)-1=N_{\beta}(\tilde{S}, j+1)$. If $N_{\beta}(S, j+1)<$ $N_{\beta}(\tilde{S}, j+1)$, then (6) follows from (9). Thus, assume

$$
N_{\beta}(\tilde{S}, j+1)=N_{\beta}(S, j+1) .
$$

If $\beta=n-1,(9)$ and (10) give equality in (6). Suppose $\beta<n-1$. By our construction of $\tilde{B}_{j+1}$, we have

$$
N_{\beta+1}(\tilde{S}, j+1)=\alpha_{j+1}-\left(N_{0}(\tilde{S}, j+1)+\ldots+N_{\beta}(\tilde{S}, j+1)\right)
$$

and

$$
N_{\beta+2}(\tilde{S}, j+1)=\ldots=N_{n-1}(\tilde{S}, j+1)=0 .
$$

Since $\operatorname{card}\left(B_{j+1}\right) \leq \alpha_{j+1}$,

$$
\begin{aligned}
& N_{\beta+1}(S, j+1) \leq \alpha_{j+1}-\left(N_{0}(S, j+1)+\ldots+N_{\beta}(S, j+1)\right) \\
= & \alpha_{j+1}-\left(N_{0}(\tilde{S}, j+1)+\ldots+N_{\beta}(\tilde{S}, j+1)\right)=N_{\beta+1}(\tilde{S}, j+1)
\end{aligned}
$$

(by (11)). Again, if $N_{\beta+1}(S, j+1)<N_{\beta+1}(\tilde{S}, j+1)$, then (6) follows from (9) and (10). So, suppose

$$
N_{\beta+1}(S, j+1)=N_{\beta+1}(\tilde{S}, j+1) .
$$

Then $N_{0}(S, j+1)+\ldots+N_{\beta+1}(S, j+1)=N_{0}(\tilde{S}, j+1)+\ldots+N_{\beta+1}(\tilde{S}, j+1)=\alpha_{j+1}$, and therefore

$$
N_{\beta+2}(S, j+1)=\ldots=N_{n-1}(S, j+1)=0 .
$$

In view of (12), we conclude that we have equality in (6).

Case 2. $N(\tilde{S}, j)>N(S, j)$ : In this case we shall show that

$$
N(\tilde{S}, j+1)>N(S, j+1) .
$$

Our hypothesis implies that there is an $\alpha \in\{0, \ldots, n-1\}$ such that

$$
N_{0}(\tilde{S}, j)=N_{0}(S, j), \ldots, N_{\alpha-1}(\tilde{S}, j)=N_{\alpha-1}(S, j) \quad \text { and } \quad N_{\alpha}(\tilde{S}, j)>N_{\alpha}(S, j) .
$$

In particular, $N_{\alpha}(\tilde{S}, j) \neq 0$, and therefore

$$
N_{i}(\tilde{S}, j+1)=N_{i}(\tilde{S}, j)=N_{i}(S, j) \quad \text { for } i=0, \ldots, \alpha-1 .
$$

Suppose that for some $i \in\{1, \ldots, \alpha\}$ we have $N_{i}(S, j+1)>N_{i}(S, j)$. Then, in the construction of $B_{j+1}$, at least one piece of $B_{j}$ with depth $i-1$ must have been replaced by subpieces. Let $i_{0} \in\{0, \ldots, \alpha-1\}$ be the smallest integer such that some piece of $B_{j}$ with depth $i_{0}$ was replaced by subpieces. Then, $N_{i}(\tilde{S}, j+1)=N_{i}(S, j) \geq$ $N_{i}(S, j+1)$ for $i=0, \ldots, i_{0}-1$, and $N_{i_{0}}(\tilde{S}, j+1)=N_{i_{0}}(S, j)>N_{i_{0}}(S, j+1)$ (by (14)), and so (13) holds. Thus, assume that

$$
N_{i}(S, j+1) \leq N_{i}(S, j) \text { for } i=1, \ldots, \alpha .
$$

If for some $i \in\{0, \ldots, \alpha-1\}$ we have $N_{i}(S, j+1)<N_{i}(S, j)$, then (13) holds (by (14) and (15)). Hence, assume

$$
N_{i}(S, j+1)=N_{i}(S, j) \quad \text { for } i=0, \ldots, \alpha-1 .
$$

By (14) and (16),

$$
N_{i}(\tilde{S}, j+1)=N_{i}(S, j+1) \quad \text { for } i=0, \ldots, \alpha-1 .
$$


Now, if some piece of $\tilde{B}_{j}$ has depth $>\alpha$, then

$$
N_{\alpha}(\tilde{S}, j+1)=N_{\alpha}(\tilde{S}, j)>N_{\alpha}(S, j) \geq N_{\alpha}(S, j+1)
$$

(by (15)), and so (13) follows from (17). Suppose that the maximum depth of the pieces in $\tilde{B}_{j}$ is exactly equal to $\alpha$. Then

$$
N_{\alpha}(\tilde{S}, j+1)=N_{\alpha}(\tilde{S}, j)-1 \geq N_{\alpha}(S, j) \geq N_{\alpha}(S, j+1) .
$$

If $N_{\alpha}(\tilde{S}, j+1)>N_{\alpha}(S, j+1)$, then (13) follows from (17). So, assume

$$
N_{\alpha}(\tilde{S}, j+1)=N_{\alpha}(S, j+1) .
$$

Hence, $N_{\alpha}(S, j)=N_{\alpha}(S, j+1)$, and so we conclude from (16) that $\alpha<n-1$ and that

$$
N_{\alpha+1}(S, j+1) \leq N_{\alpha+1}(S, j) .
$$

Now, the fact that $\tilde{B}_{j}$ has no piece with depth $>\alpha$ implies that

$$
N_{\alpha+1}(\tilde{S}, j+1)=\alpha_{j+1}-\left(N_{0}(\tilde{S}, j+1)+\ldots+N_{\alpha}(\tilde{S}, j+1)\right) .
$$

Consequently,

$$
\begin{aligned}
& N_{\alpha+1}(S, j+1) \leq N_{\alpha+1}(S, j) \leq \alpha_{j}-\left(N_{0}(S, j)+\ldots+N_{\alpha}(S, j)\right) \\
& <\alpha_{j+1}-\left(N_{0}(\tilde{S}, j+1)+\ldots+N_{\alpha}(\tilde{S}, j+1)\right)=N_{\alpha+1}(\tilde{S}, j+1) .
\end{aligned}
$$

In view of (17) and (18), we conclude that (13) holds. This completes the proof of lemma 4.

Remark 5. The above proof shows that if $S=\left(B_{1}, \ldots, B_{r}\right) \in \Omega$ and if $N(\tilde{S}, j)>$ $N(S, j)$ for some $j \in\{0, \ldots, \min \{M, r\}\}$, then

$$
N(\tilde{S}, t)>N(S, t) \quad \text { for all } t \in\{j, \ldots, \min \{M, r\}\} .
$$

In particular, $r<M$. This shows that the sequences $S \in \Omega$ that have length $M$ are exactly those that satisfy the condition

$$
N(S, j)=N(\tilde{S}, j) \quad \text { for all } j \in\{0, \ldots, M\} .
$$

\section{Further RESUlts}

We now remark that the argument used to prove theorem 1 can also be applied to establish other results of similar type. For instance, we have the following

Theorem 6. Let $n$ and $d$ be positive integers. Then there is a constant $L=$ $L_{\mathbf{K}}(n, d) \in \mathbf{N}^{*}$ such that for any homogeneous polynomial maps $P, Q: \mathbf{K}^{n} \rightarrow \mathbf{K}^{n}$ of degree $d$ and any $x, y \in \mathbf{K}^{n}$, the relation

$$
\left\|P^{j} x\right\|=\left\|Q^{j} y\right\| \text { for } j=1, \ldots, L
$$

implies

$$
\left\|P^{j} x\right\|=\left\|Q^{j} y\right\| \text { for all } j \geq 1 .
$$

Proof. We may assume $\mathbf{K}=\mathbf{R}$. Now, we have to consider the homogeneous polynomial $q_{j}: \mathbf{R}^{2 n} \rightarrow \mathbf{R}$ given by

$$
q_{j}(z, w)=\left\langle P^{j} z, P^{j} z\right\rangle-\left\langle Q^{j} w, Q^{j} w\right\rangle \quad\left(z, w \in \mathbf{R}^{n}\right) .
$$

As before, we define

$$
\begin{aligned}
& A_{j}=\left\{(z, w) \in \mathbf{R}^{2 n} ; q_{k}(z, w)=0 \text { for } k=1, \ldots, j\right\} \\
& =\left\{(z, w) \in \mathbf{R}^{2 n} ;\left\|P^{k} z\right\|=\left\|Q^{k} w\right\| \text { for } k=1, \ldots, j\right\}
\end{aligned}
$$


and consider the algebraic subset

$$
Y_{j}=Z\left(\tilde{q}_{1}\right) \cap \ldots \cap Z\left(\tilde{q}_{j}\right)
$$

of $\mathbf{P}^{2 n-1}$. The same argument used to prove theorem 1 then applies (the $\alpha_{j}$ 's are the same, but we have to consider $2 n$ in place of $n$ ).

Similarly, we obtain

Theorem 7. Let $n$ and $d$ be positive integers. Then there is a constant $R=$ $R_{\mathbf{K}}(n, d) \in \mathbf{N}^{*}$ such that for any homogeneous polynomial maps $P, Q: \mathbf{K}^{n} \rightarrow \mathbf{K}^{n}$ of degree $d$ and any $x, y \in \mathbf{K}^{n}$, the relation

$$
1=\|x-y\|=\|P x-Q y\|=\ldots=\left\|P^{R} x-Q^{R} y\right\|
$$

implies

$$
\left\|P^{j} x-Q^{j} y\right\|=1 \text { for all } j \geq 1 .
$$

Remark 8. Note that theorem 7 generalizes theorem 1 (just set $Q=P$ and $y=0$ ).

We close the paper by proposing the following question:

Open problem. What are the best values of the constants $M_{\mathbf{R}}(n, d), M_{\mathbf{C}}(n, d)$, $N_{\mathbf{R}}(n, d)$ and $N_{\mathbf{C}}(n, d)$ ?

Only the following are known: $N_{\mathbf{R}}(n, 1)=N_{\mathbf{C}}(n, 1)=n$ (see [4]), $M_{\mathbf{R}}(1,1)=$ $M_{\mathbf{C}}(1,1)=1, M_{\mathbf{R}}(2,1)=3, M_{\mathbf{C}}(2,1)=4$ and $M_{\mathbf{C}}(3,1)=8$ (see [1]). Moreover, some estimates for $M_{\mathbf{R}}(n, 1)$ and $M_{\mathbf{C}}(n, 1)$ were obtained in [1].

\section{ACKNOWLEDGMENT}

The present work is part of the author's Ph.D. Thesis at Kent State University. The subject of this work was suggested by Professor Per Enflo, to whom the author is deeply grateful. The author would like also to thank CAPES (Brazil) for financial support.

\section{REFERENCES}

[1] P. Enflo, V. I. Gurarii, V. Lomonosov and Yu. I. Lyubich, Exponential numbers of linear operators in normed spaces, Linear Algebra Appl. 219 (1995), 225-260. MR 96c: 47026

[2] W. Fulton, Intersection Theory, Springer-Verlag, 1984. MR 85k:14004

[3] R. Hartshorne, Algebraic Geometry, Springer-Verlag, 1977. MR 57:3116

[4] V. Pták, Norms and the spectral radius of matrices, Czechoslovak Math. J. 12 (1962), 555557. MR 26:3712

Instituto de Matemática, Universidade Federal Fluminense, Rua Mário Santos Braga S/N, 24020-140, Niterói, RJ, BrasiL

E-mail address: ganncbj@vm.uff.br 